\title{
Disposition of Methamphetamine and Major Metabolites in Mice: Role of Organic Cation Transporter 3 in Tissue-Selective Accumulation of Para-Hydroxymethamphetamine
}

\author{
David J. Wagner, Laura M. Shireman, Sojung Ahn, Danny D. Shen, and Joanne Wang \\ Departments of Pharmaceutics (D.J.W., L.M.S., D.D.S., J.W.) and Pharmacy (S.A.), University of Washington, Seattle, Washington \\ Received April 18, 2018; accepted June 12, 2018
}

\begin{abstract}
Methamphetamine is one of the most widely abused illicit drugs. Although human intoxication and multiple tissue toxicities frequently occur in abusers, little is known about the distribution of methamphetamine or its primary metabolites, amphetamine and parahydroxymethamphetamine ( $p$-OHMA), to their sites of toxicity. This study determined the pharmacokinetics, tissue exposure, and partition ratios of methamphetamine and major metabolites in various mouse tissues and investigated the impact of organic cation transporter 3 (Oct3) following i.v. injection of methamphetamine to male $\mathrm{Oct}^{+/+}$and $\mathrm{Oct3}^{-/-}$mice. Methamphetamine, amphetamine, and $p$-OHMA were readily detectable in plasma with $\mathrm{Oct}^{+/+}$and Oct $^{-1-}$ mice displaying similar plasma pharmacokinetic profiles for all three analytes. In addition to kidney and liver, salivary glands highly accumulated methamphetamine, amphetamine, and $p$-OHMA
\end{abstract}

with total exposure 3.3- to 9.4-fold higher than plasma area under the concentration-time curve (AUC). Consistent with being an Oct3 substrate, $p$-OHMA AUC in salivary glands is reduced by $50 \%$ in Oct $^{-1-}$ mice. $p$-OHMA AUC in skeletal muscle is also significantly reduced in $\mathrm{Oct}^{-1-}$ mice. Our data identified salivary glands as a novel site of high accumulation of methamphetamine and metabolites, which may underlie methamphetamine toxicity in this tissue. Furthermore, our study identified Oct3 as an important determinant of tissue uptake and exposure to $p$-OHMA in salivary glands and skeletal muscle. Our findings suggest that local tissue accumulation of methamphetamine and/or its metabolites may play a role in several of the reported peripheral toxicities of methamphetamine, and Oct3 can significantly impact tissue exposure to its substrates without affecting systemic elimination.

\section{Introduction}

Methamphetamine is one of the most widely abused and toxic illicit drugs (Volkow et al., 2010; Volkow, 2013). Methamphetamine, or meth, is a potent and highly addictive central nervous stimulant that acts by inhibiting and reversing the dopamine transporter, norepinephrine transporter, and serotonin transporter (Carvalho et al., 2012; Panenka et al., 2013). In vivo, methamphetamine is metabolized to two major primary metabolites, amphetamine and para-hydroxymethamphetamine (p-OHMA) (Lin et al., 1997; Shima et al., 2008). Amphetamine has similar psychoactive and addictive properties as methamphetamine (Carvalho et al., 2012; Panenka et al., 2013). p-OHMA is not psychoactive but has cardiovascular activity with hypertensive and adrenergic activity (Römhild et al., 2003).

Methamphetamine is considered one of the most toxic drugs of abuse with both central nervous system and peripheral toxicities (Volkow et al., 2010). Methamphetamine neurotoxicity is relatively well understood with long-term neurologic injuries primarily targeting the

This work was supported by the National Institutes of Health National Institute on Drug Abuse [Grant P01 DA032507] and National Institutes of Health General Medical Sciences [Grant T32 GM07750]. The content is solely the responsibility of the authors and does not necessarily represent the official views of the National Institutes of Health.

https://doi.org/10.1124/dmd.118.082131. dopaminergic and serotonergic neurons, where methamphetamine is highly concentrated by the monoamine transporters (Schep et al., 2010; Panenka et al., 2013). The peripheral toxicity colloquially known as meth mouth, xerostomia (dry mouth) and rampant dental caries, is one of the most widely known injuries that is publicized in many antidrug campaigns (Shaner et al., 2006). Little is currently known regarding the mechanisms leading to meth mouth. It has been speculated that methamphetamine reduces saliva flow through vasoconstriction of the capillaries around salivary glands (Hamamoto and Rhodus, 2009). However, a recent study in abusers indicated that xerostomia was not caused by a decreased saliva output but rather a change in saliva composition, suggesting direct toxicity to salivary glands (Ravenel et al., 2012). In addition, methamphetamine abuse is associated with muscle toxicities. In severe cases, a breakdown of muscle leads to rhabdomyolysis and injury of multiple organs (Carvalho et al., 2012).

Elimination of methamphetamine is by both hepatic metabolism and renal secretion with approximately $40 \%$ excreted unchanged in the urine (Kim et al., 2004). The polymorphic enzyme cytochrome P450 2D6 is the major liver enzyme that metabolizes methamphetamine to the primary circulating metabolites amphetamine and p-OHMA (Lin et al., 1997; Shima et al., 2008). We previously showed that methamphetamine and metabolites inhibit human organic cation transporters (hOCTs) and multidrug and toxin extrusion transporters and are selectively transported by these transporters (Wagner et al., 2017). Renal excretion is another major elimination pathway for methamphetamine and its metabolites. We previously demonstrated that

ABBREVIATIONS: AUC, area under the concentration-time curve; HBSS, Hanks' balanced salt solution; HEK, human embryonic kidney; hOCT, human OCT; LC-MS/MS, liquid chromatography tandem mass spectrometry; mOct, murine Oct; OCT, organic cation transporter; PET, positron emission tomography; $p$-OHMA, para-hydroxymethamphetamine. 
methamphetamine, amphetamine, and $p$-OHMA are all substrates of the renal hOCT2 and multidrug and toxin extrusion transporters in vitro, suggesting that these transporters may play a role in the renal handling of methamphetamine and metabolites (Wright, 2005; Wagner et al., 2017). Organic cation transporter (OCT)1, the major isoform in the liver, may be involved in the hepatic handling of $p$-OHMA as it is transported by hOCT1 in vitro; however, methamphetamine and amphetamine are not substrates (Wagner et al., 2017).

Animal studies and human positron emission tomography (PET) studies showed that methamphetamine and metabolites are distributed into many organs with high accumulations (Rivière et al., 2000; Volkow et al., 2010). Norepinephrine transporter- and dopamine transportermediated high accumulation of methamphetamine and amphetamine in neuronal tissues is thought to at least partially contribute to neurotoxicity (de la Torre et al., 2004; Carvalho et al., 2012). In contrast, the role of transporters in peripheral distribution is poorly understood in vivo (Panenka et al., 2013). As cations with reported or calculated LogD values of $-0.38,-0.62$, and -1.11 , methamphetamine, amphetamine, and $p$-OHMA may have low passive permeability as protonated species (Fowler et al., 2007). We and others have reported that OCT3 is highly expressed in a number of tissues, including salivary glands and skeletal muscle in both human and rodents (Koepsell et al., 2007; Lee et al., 2013, 2014; Chen et al., 2015). Using an Oct3 knockout mouse model, we previously showed that OCT3 is responsible for the accumulation and secretion of metformin (an organic cation drug) in salivary glands (Lee et al., 2014). Recently, we identified $p$-OHMA is an in vitro substrate of hOCT3, suggesting that OCT3 may play a role in tissuespecific accumulation of $p$-OHMA (Wagner et al., 2017).

The goals of this study were to characterize the distribution of methamphetamine and its primary circulating metabolites amphetamine and $p$-OHMA in mice and determine the in vivo role of OCT3 in tissue disposition. We determined the pharmacokinetics and tissue partitioning of methamphetamine and metabolites in mice. The in vivo significance of Oct3 in methamphetamine, amphetamine, and p-OHMA tissue distribution was evaluated using a mouse model with a targeted deletion of the Oct3/Slc22a3 gene.

\section{Materials and Methods}

Materials. Analytical grade $d$-methamphetamine, $d$-amphetamine, $p$-OHMA, and ammonium formate were purchased from Sigma-Aldrich (St. Louis, MO). The $d$-methamphetamine isomer is the primary psychoactive entity, although racemic methamphetamine is produced by some synthesis methods (de la Torre et al., 2004). We used the dextro isoform of methamphetamine in this study because it is the psychoactive form. Methamphetamine- $\mathrm{D}_{11}$ and amphetamine$\mathrm{D}_{11}$ were purchased from Cerilliant (Round Rock, TX). $\left[{ }^{14} \mathrm{C}\right]$ Metformin $(115 \mathrm{mCi} / \mathrm{mmol})$ was purchased from Moravek Biochemicals (Brea, CA). Optima grade acetonitrile, methanol, water, and formic acid were purchased from Fisher Scientific (Waltham, MA). Cell culture media and supplements were purchased from Invitrogen (Carlsbad, CA). All other chemicals were commercially available and of analytical grade or higher.

Animals. Null Oct3 (Slc22a3) mice were originally developed from the FVB inbred strain by Dr. Denise Barlow (Zwart et al., 2001) and maintained by Dr. Alfred Schinkel (Netherlands Cancer Institute). Breeding pairs of both wildtype $\mathrm{Oct}^{+/+}$and $\mathrm{Oct}^{-/-}$mice were generously provided by Dr. John Markowitz at the University of Florida (Gainesville, FL) after rederivation at Charles River Laboratories (Wilmington, MA) (Zhu et al., 2010). Mice were housed in specific pathogen-free facilities at the University of Washington with a 14/10-hour light/dark cycle and a standard diet. All animal studies were approved by the Institutional Animal Care and Use Committee of the University of Washington.

Uptake Experiments. Flp-in human embryonic kidney (HEK)293 cells stably expressing murine Oct (mOct) 3 were previously generated in our laboratory (Lee et al., 2014). The cells were cultured in high-glucose Dulbecco's modified Eagle's medium with $10 \%$ fetal bovine serum, $1 \mathrm{mM} \mathrm{L}$-glutamine, $100 \mathrm{U} / \mathrm{ml}$ penicillin, $100 \mu \mathrm{g} / \mathrm{ml}$ streptomycin, and $150 \mu \mathrm{g} / \mathrm{ml}$ hygromycin B supplementation at $37^{\circ} \mathrm{C}$ with $5 \% \mathrm{CO}_{2}$ and high humidity. Cell culture flasks and plates were coated with $0.01 \%$ poly-D-lysine to improve cell attachment. Uptake assays were performed, as previously described (Wagner et al., 2017). Briefly, cells were seeded in 96-well plates at 100,000 cells/well and grown overnight. Cells were washed with prewarmed Hanks' balanced salt solution (HBSS) and allowed to acclimate for 10 minutes at $37^{\circ} \mathrm{C}$ prior to uptake experiments. HBSS was removed, and incubations were initiated by the addition of $100 \mu 1 \mathrm{HBSS}$ at $\mathrm{pH} 7.4$ containing substrate. Incubations were terminated by removing media and washing the cells three times with ice-cold HBSS. Metformin, used as a reference substrate, was measured by liquid scintillation counting (Tri-Carb B3110TR; PerkinElmer, Waltham, MA) after lysis with $100 \mu 11 \mathrm{M} \mathrm{NaOH}$ for 1 hour and neutralization with $100 \mu \mathrm{l} 1 \mathrm{M} \mathrm{HCl}$. Methamphetamine, amphetamine, and $p$-OHMA were quantified by liquid chromatography tandem mass spectrometry (LC-MS/MS), as described below. Briefly, cells were permeabilized with $100 \mu \mathrm{l} /$ well methanol containing $100 \mathrm{nM}$ stable-labeled internal standards for 15 minutes, followed by dilution into an equal volume of Optima water. Uptake was normalized to total protein measured in the lysate by the bicinchoninic acid protein assay kit (Pierce Chemical, Rockford, IL). Kinetic experiments were performed during the initial rate period using a short incubation time (1 minute). Uptake experiments were performed in triplicate and repeated independently three times. Transporter-specific uptake was calculated by subtracting uptake in vectortransfected cells from uptake in the mOct3-transfected cells. Transport experiments were performed in triplicate and repeated three independent times. Data representation and replicates with specific numbers of observations are detailed in each figure legend. Because the Eadie-Hofstee plot indicated cooperativity, mOct3 transporter kinetics of $p$-OHMA were fitted to the Michaelis-Menten equation with a Hill slope $(\mathrm{H})$ to obtain half-maximal transport concentration $\left(\mathrm{K}_{1 / 2}\right.$ in place of Michaelis-Menten constant) and maximal transport rate $\left(\mathrm{V}_{\max }\right)$ using GraphPad Prism 6.0 (GraphPad Software, La Jolla, CA) (Copeland, 2000):

$$
\mathrm{v}=\frac{\mathrm{V}_{\max } * \mathrm{~S}^{\mathrm{H}}}{\mathrm{K}_{1 / 2}{ }^{\mathrm{H}}+\mathrm{S}^{\mathrm{H}}}
$$

In Vivo Pharmacokinetics Studies. All in vivo studies were carried out in male FVB mice 10-12 weeks old. Only male mice were used, as we and others previously observed similar pharmacokinetics and tissue distribution of organic cations, including amphetamine, between male and female mice (Zhu et al., 2010; Lee et al., 2014). Mice were administered $10 \mathrm{mg} / \mathrm{kg}$ methamphetamine i.v. by retro-orbital injection. This dose did not induce acute toxicity in mice and generated plasma concentrations of methamphetamine and metabolites that are within the ranges reported in methamphetamine abusers (Shima et al., 2008). Mice ( $n=3-6$ at each time point) were sacrificed by cardiac puncture with a heparinized syringe under isoflurane anesthesia at various time points $(2,15,30$, $60,180,300$, and 480 minutes). Blood was centrifuged at $1700 \mathrm{~g}$, and the plasma was stored at $-80^{\circ} \mathrm{C}$ until analysis. Tissues (submandibular salivary glands, brain, heart, liver, kidney, skeletal muscle, and adipose) were collected at each time point, snap frozen, and stored at $-80^{\circ} \mathrm{C}$ until use. Methamphetamine, amphetamine, and $p$-OHMA concentrations in plasma and tissue homogenates were determined by LC-MS/MS analysis. Concentrations were expressed as nanomolar for plasma and tissue assuming a density of $1 \mathrm{~g} / \mathrm{ml}$.

LC-MS/MS Analysis of Methamphetamine and Its Metabolites. Methamphetamine, amphetamine, and $p$-OHMA were quantified using a LC-MS/MS method adapted from our previously established procedures (Wagner et al., 2017). In vitro samples were prepared by permeabilization of cells with $100 \mu 1$ methanol containing $100 \mathrm{nM}$ stable-labeled internal standard and dilution into an equal volume of Optima water. Tissue samples were homogenized with 1:2 weight: volume phosphate-buffered saline by an Omni Bead Ruptor (Omni International, Kennesaw, GA). Tissue density was assumed to be $1 \mathrm{~g} / \mathrm{ml}$. In vivo plasma and tissue samples were prepared by protein precipitation with 5 volumes of $75 \%$ acetonitrile/25\% methanol containing $100 \mathrm{nM}$ internal standards, followed by centrifugation at $(20,000 \mathrm{~g})$, evaporation under nitrogen, and reconstitution in $50 \%$ methanol in water. The LC-MS/MS system consisted of an AB-Sciex 5500 qTrap Q-LIT mass spectrometer (Foster City, CA) coupled with an Agilent 1290 ultra performance liquid chromatography (UPLC) (Santa Clara, CA) and a Phenomenex Synergi Hydro-RP column $(50 \times 2 \mathrm{~mm} ; 2.5 \mu \mathrm{m})$. The Turbo Ion Spray interface was operated in positive ion mode. Gradient elution at $0.5 \mathrm{ml} / \mathrm{min}$ with $5 \mathrm{mM}$ ammonium formate in water at $\mathrm{pH} 3(\mathrm{~A})$ and acetonitrile (B) was as follows: 
$10 \%$ B until 0.1 minute, increased to $50 \%$ B by 1.5 minutes, then to $95 \%$ B by 1.51 minutes, and held at $95 \% \mathrm{~B}$ until 2 minutes, returning to $10 \% \mathrm{~B}$ at 2.1 minutes with a total run time of 3 minutes. Mass transitions $(\mathrm{m} / \mathrm{z})$ for methamphetamine, amphetamine, $p$-OHMA, methamphetamine- $\mathrm{d}_{11}$, and amphetamine- $d_{11}$ were $150 \rightarrow 119,136 \rightarrow 91,166 \rightarrow 135,161 \rightarrow 97$, and $147 \rightarrow 98$, respectively. Data were analyzed with Analyst software version 1.6.3 (AB Sciex). Assay accuracy and precision were within $15 \%$ (20\% for the lower limit of quantification).

Pharmacokinetic Data Analysis. The mean and S.E. for pharmacokinetic parameters were estimated using a population-based bootstrap method previously described due to sampling plasma and tissue from different animals at each time point (Mager and Göller, 1998; Lee et al., 2014). Briefly, pseudo concentrationtime data were created using the R package PK (RStudio version 1.1.383 with $\mathrm{R}$ version 3.4.2) (Jaki and Wolfsegger, 2011; RStudio, 2012; R Core Team, 2017) by resampling measured plasma or tissue concentrations 10,000 times with random replacement of individual animals. The pharmacokinetic parameters were determined with a noncompartmental approach using the equations defined below. The area under the concentration-time curve (AUC) was calculated using the linear trapezoidal rule. To normalize tissue AUC to any variability in systemic AUC, AUC ratios were calculated by dividing each tissue $\mathrm{AUC}_{0-480 \text { min }}$ by the plasma $\mathrm{AUC}_{0-480 \text { min }}$ as an estimate of tissue partition ratios.

The following equations were used to calculate exposure (AUC), clearance (CL), terminal half-life $\left(t_{1 / 2, \beta}\right)$, and volume of distribution at steady state $\left(V_{\mathrm{ss}}\right)$ :

$$
\begin{aligned}
\mathrm{AUC}_{0-\mathrm{t}} & =\int_{0}^{\mathrm{t}} \mathrm{C}(\mathrm{t}) \mathrm{dt} \\
\mathrm{AUC}_{0-\infty} & =\mathrm{AUC}_{0-\mathrm{t}}+\frac{\mathrm{C}(\mathrm{T})}{\beta} \\
\mathrm{CL} & =\frac{\text { Dose }}{\mathrm{AUC}_{0-\infty}} \\
\mathrm{t}_{\frac{1}{2}, \beta} & =\frac{\ln 2}{\beta} \\
\mathrm{V}_{\mathrm{ss}} & =\frac{\text { Dose* AUMC }^{*}}{\mathrm{AUC}^{2}}
\end{aligned}
$$

The terminal slope $(\beta)$ was calculated by linear regression of log concentrations of the terminal phase of the concentration-time profile.

Statistical Analysis. An unpaired Student's $t$ test with multiple tests corrected by the Bonferroni method was used for in vitro studies to calculate $P$ values. S.E. around the point estimate for each pharmacokinetic parameter were determined using the nonparametric bootstrap method (Efron and Tibshirani, 1994; Jaki and Wolfsegger, 2011; Lee et al., 2014). Two-sided $P$ values for pharmacokinetic parameters were calculated using permutation tests, as described previously (Westfall and Young, 1993; Jaki and Wolfsegger, 2011; Lee et al., 2014). Multiple comparisons were corrected using the Bonferroni method. A $P$ value $<0.05$ was considered statistically significant.

\section{Results}

Transport of Methamphetamine and Metabolites by mOct3 Stably Expressed in HEK293 Cells. We previously demonstrated that hOCT3 shows substrate specificity for amphetamines by transporting p-OHMA, but not amphetamine or methamphetamine (Wagner et al., 2017). To confirm mOct 3 demonstrates similar substrate specificity, methamphetamine, amphetamine, and $p$-OHMA were screened for uptake in mOct3 and empty vector-transfected cells (Fig. 1A). Similar to hOCT3, only $p$-OHMA demonstrated preferential uptake into mOct3 cells when compared with vector-transfected cells (Fig. 1, A and B). Initial rate uptake studies showed that mOct3-mediated transport of p-OHMA was sigmoidal (Fig. 1C), and the cooperativity was more clearly revealed by the Eadie-Hofstee plot (Fig. 1D). The half-maximal transport concentration $\left(\mathrm{K}_{1 / 2}\right)$ is $120 \pm 26 \mu \mathrm{M}$, and the maximal transport rate $\left(\mathrm{V}_{\max }\right)$ is $3100 \pm 1000 \mathrm{pmol} / \mathrm{min}$ per milligram total protein. The fitted Hill slope is $1.29 \pm 0.15$, suggesting a mild cooperativity.

Methamphetamine Pharmacokinetic Studies in Oct $^{+/+}$and oct $^{-1-}$ Mice. To determine the in vivo significance of OCT3 in the disposition of methamphetamine and metabolites, we determined plasma and tissue concentrations of methamphetamine, amphetamine, and $p$-OHMA in $O c t 3^{+/+}$and $O c t 3^{-/-}$mice following i.v. injection of $10 \mathrm{mg} / \mathrm{kg}$ methamphetamine. The plasma concentration-time profiles for methamphetamine, amphetamine, and $p$-OHMA in Oct $^{+/+}$and $\mathrm{Oct}^{-1-}$ mice are shown in Fig. 2, with plasma methamphetamine pharmacokinetic parameters summarized in Table 1 . The metabolite p-OHMA was rapidly observed in plasma, with its peak concentration at the 2-minute time point. Conversely, amphetamine reached peak plasma concentrations at approximately 60 minutes. Plasma clearance, exposure, terminal half-life, and volume of distribution were comparable for methamphetamine in $\mathrm{Oct}^{+/+}$and $\mathrm{Oct}^{-/-}$mice (Table 1). Amphetamine and $p$-OHMA also displayed similar plasma AUCs in both $O c t 3^{+/+}$and Oct $3^{-1-}$ mice (Fig. 2). The plasma concentrations and overall exposure of the two metabolites in both genotypes are much lower than the parent drug (Fig. 2; Table 2). These data suggest that Oct3 does not play a significant role in systemic exposure to methamphetamine and major metabolites following i.v. injection.

Impact of Oct3 Deletion on Salivary Glands Exposure to Methamphetamine, Amphetamine, and p-OHMA. To determine the in vivo significance of OCT3 in the salivary glands distribution of methamphetamine and metabolites, salivary glands were collected at each time point in the pharmacokinetic study, and concentrations were
A

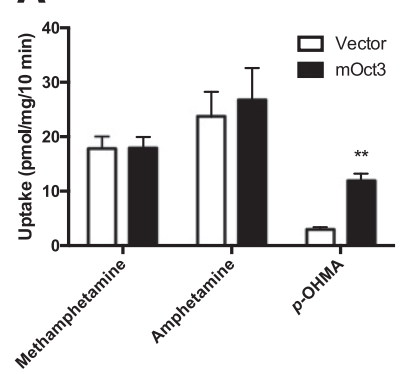

B

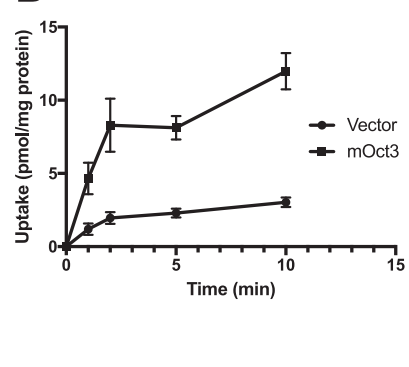

C

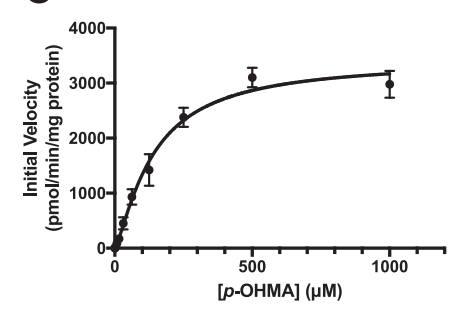

D

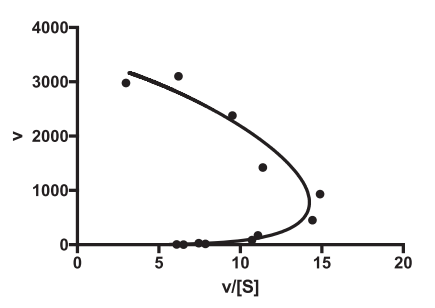

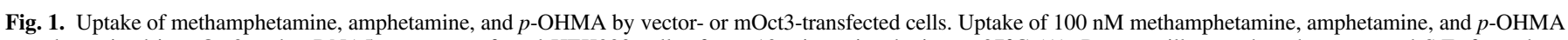

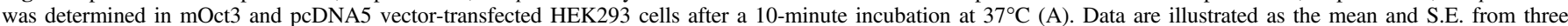

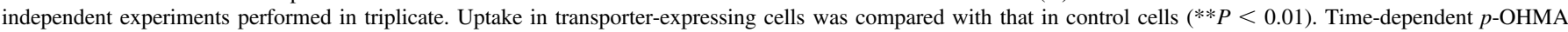

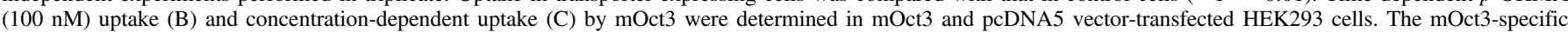

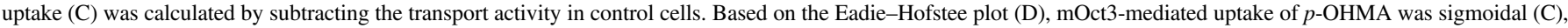

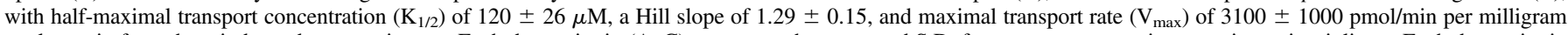

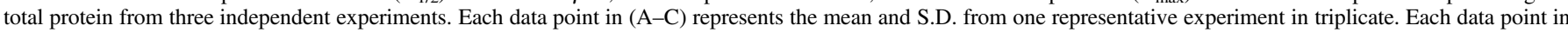
(D) represents the mean transformed value; no error bar is shown due to uneven error propagation. 
A
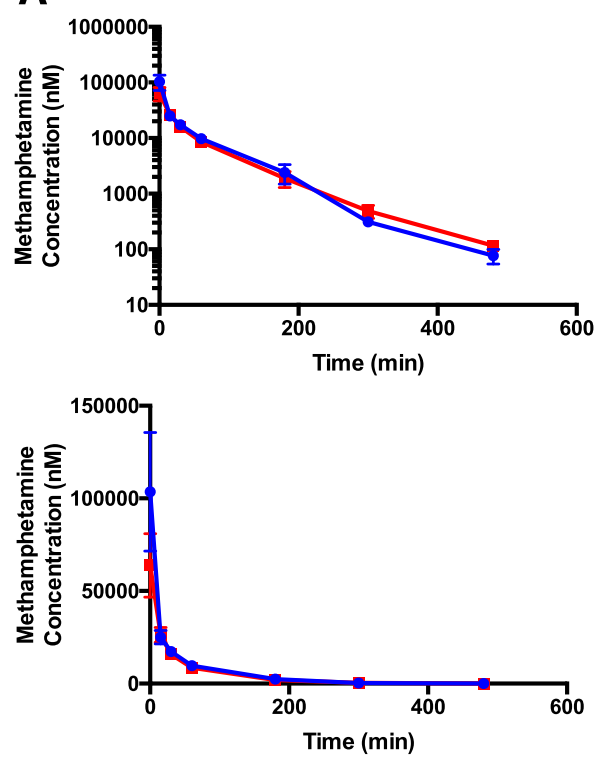

B

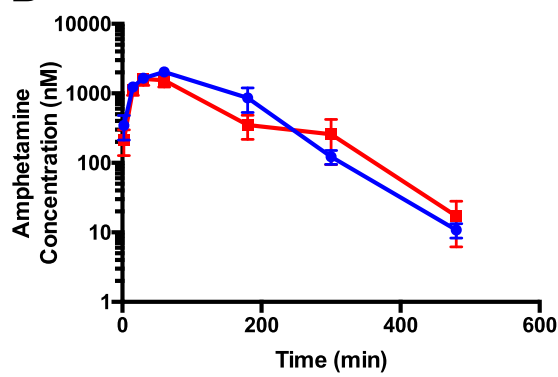

C

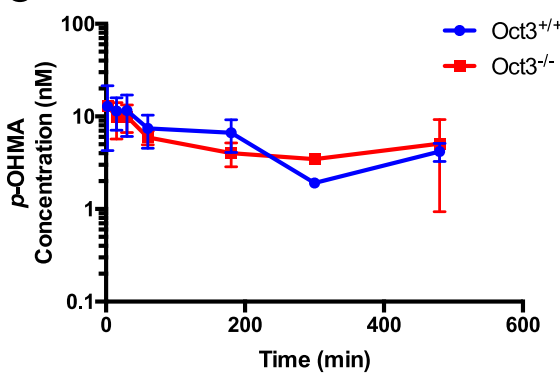

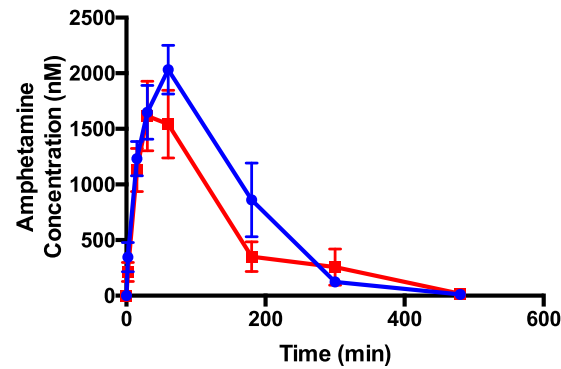

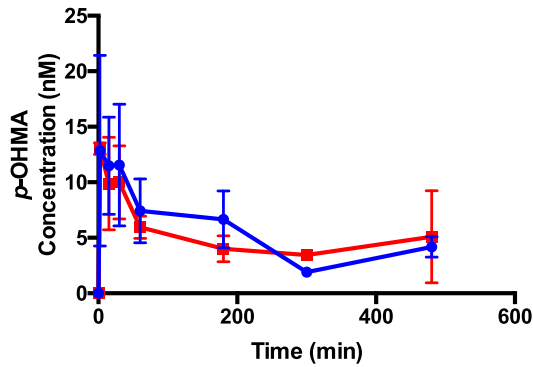

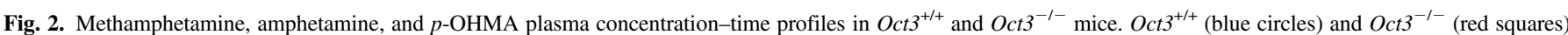

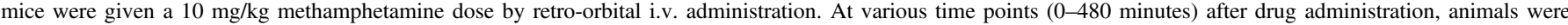

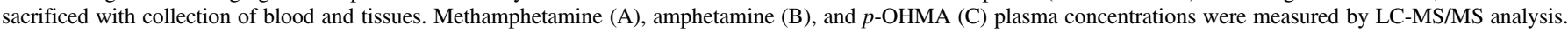

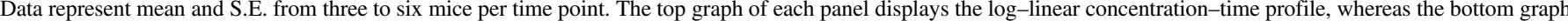
displays the linear-linear profile.

determined in tissue homogenate (Fig. 3). As we expected, we did not observe any significant genotype-dependent differences in salivary glands exposure for methamphetamine or amphetamine. In contrast, knockout mice had consistently lower concentrations of $p$-OHMA (Fig. 3) in salivary glands with approximately a 50\% reduction in salivary tissue AUC when compared with Oct $^{+/+}$mice (Table 2). These data suggest $p$-OHMA is actively transported by Oct 3 from the blood into the salivary glands. The importance of Oct3 in salivary glands uptake of $p$-OHMA in vivo was further reflected by its high partitioning ratio in salivary glands of $\operatorname{Oct}^{+/+}$mice $(9.4 \pm 1.9)$, which was greatly reduced in the knockout mice $(4.5 \pm 0.89)$ (Table 3$)$.

Tissue Distribution of Methamphetamine, Amphetamine, and p-OHMA. To characterize the tissue distribution of methamphetamine and major metabolites, various tissues (muscle, brain, heart, adipose, liver, and kidney) were collected at each time point in the pharmacokinetic study. Methamphetamine, amphetamine, and $p$-OHMA in various tissue homogenates were quantified by LC-MS/MS, and the overall exposure ( $\mathrm{AUC}_{0-480 \mathrm{~min}}$ ) was determined (Fig. 4A; Table 2). The tissue partitioning ratio was calculated by normalizing tissue $\mathrm{AUC}_{0-480}$ min to plasma $\mathrm{AUC}_{0-480 \mathrm{~min}}$ (Fig. 4B; Table 3). For methamphetamine and amphetamine, the kidney, liver, and brain had the highest exposure, with tissue AUCs approximately 4-20 times higher than the plasma

TABLE 1

Methamphetamine plasma pharmacokinetic parameters in $O c t 3^{+/+}$and $O c t 3^{-/-}$mice

Data are presented as mean \pm S.E. No statistically significant differences were determined between genotypes for each pharmacokinetic parameter using a permutation test with multiple comparisons corrected using the Bonferroni method, as detailed in Materials and Methods.

\begin{tabular}{lcc}
\hline \multicolumn{1}{c}{ Parameters } & Oct $^{+/+}$ & Oct $^{-/-}$ \\
\hline $\mathrm{AUC}_{\text {inf }}(\mu \mathrm{M} \times \mathrm{min})$ & $2600 \pm 270$ & $2200 \pm 210$ \\
Terminal $t_{1 / 2}(\mathrm{~min})$ & $35 \pm 4.2$ & $59 \pm 5.2$ \\
Clearance $(\mathrm{ml} / \mathrm{min}$ per kilogram $)$ & $26 \pm 2.6$ & $31 \pm 3.0$ \\
$V_{\mathrm{ss}}(\mathrm{l} / \mathrm{kg})$ & $1.3 \pm 0.23$ & $1.8 \pm 0.26$ \\
\hline
\end{tabular}

$t_{1 / 2}$, half-life; $V_{\mathrm{ss}}$, volume of distribution at steady state.
AUC (Fig. 4; Tables 2 and 3). Remarkably, salivary glands also showed very high exposures to methamphetamine and amphetamine, with AUC and partitioning ratio comparable to those in organs of elimination (liver and kidney) and site of action (brain). The heart, muscle, and adipose tissues showed much lower exposures, with partitioning ratios ranging from 0.24 to 2.3 (Table 3). Consistent with methamphetamine and amphetamine not being transported by Oct3 (Fig. 1), there was no significant difference in tissue distribution of methamphetamine or amphetamine between $\operatorname{Oct}^{+/+}$and $O c t 3^{-/-}$mice.

The concentrations of $p$-OHMA in various tissues in general are much lower than methamphetamine and amphetamine. In $\mathrm{Oct}^{+/+}$mice, liver, kidney, and salivary glands showed the highest exposure, followed by brain, skeletal muscle, and heart. Adipose exposure to $p$-OHMA cannot be determined because the concentrations at most time points were below the quantification limit. In addition to salivary glands, muscle exposure to $p$-OHMA was significantly lower in $O c t 3^{-1-}$ (Figs. 3 and 4; Table 2), suggesting that Oct3 may also play a role in $p$-OHMA distribution into skeletal muscle. The mean AUC and tissue-to-plasma ratios of $p$-OHMA in the brain were $\sim 2$.6-fold higher in the $O c t 3^{+/+}$mice than in the $O c t 3^{-1-}$ mice, although the $P$ value corrected for multiple comparisons did not reach statistical significance (Tables 2 and 3). Similar -OHMA exposure was observed in the liver, kidney, and heart between $\mathrm{Oct}^{+/+}$and $\mathrm{Oct}^{-/-}$mice.

\section{Discussion}

Methamphetamine is one of the most widely abused illicit drugs. Whereas human intoxication and multiple tissue toxicities frequently occur in abusers, little is known about methamphetamine or its primary metabolites' distribution to their sites of toxicity (Volkow et al., 2010; Volkow, 2013). This study determined the pharmacokinetics, tissue exposure, and partition ratios of methamphetamine and major metabolites in various mouse tissues and investigated the impact of Oct 3 on the tissuespecific accumulation of $p$-OHMA. Our data demonstrated that salivary glands are a novel site of high accumulation of methamphetamine, 
TABLE 2

Methamphetamine, amphetamine, and $p$-OHMA tissue $\mathrm{AUC}_{0-480}(\mu \mathrm{M} \times \min )$ in $O c t 3^{+/+}$and $O c t 3^{-1-}$ mice

Data are presented as mean \pm S.E. Statistical significance was determined between genotypes for each analyte in each tissue using a permutation test with multiple comparisons corrected using the Bonferroni method, as detailed in Materials and Methods.

\begin{tabular}{|c|c|c|c|c|c|c|}
\hline \multirow{2}{*}{ Tissue } & \multicolumn{2}{|c|}{ Methamphetamine } & \multicolumn{2}{|c|}{ Amphetamine } & \multicolumn{2}{|c|}{$p$-OHMA } \\
\hline & $O c t 3^{+/+}$ & $O c t 3^{-1-}$ & Oct $3^{+/+}$ & $O c t 3^{-1-}$ & $O c t 3^{+/+}$ & $O c t 3^{-1-}$ \\
\hline Plasma & $2600 \pm 300$ & $2200 \pm 210$ & $330 \pm 44$ & $250 \pm 38$ & $2.5 \pm 0.42$ & $2.6 \pm 0.49$ \\
\hline Salivary glands & $12,000 \pm 1100$ & $9500 \pm 510$ & $1300 \pm 200$ & $1000 \pm 160$ & $24 \pm 2.7$ & $12 \pm 0.85 * *$ \\
\hline Muscle & $2500 \pm 580$ & $2600 \pm 240$ & $120 \pm 21$ & $160 \pm 28$ & $9.9 \pm 2.4$ & $2.4 \pm 0.77 *$ \\
\hline Heart & $4900 \pm 500$ & $5100 \pm 950$ & $460 \pm 44$ & $420 \pm 99$ & $7.3 \pm 1.5$ & $6.4 \pm 3.6$ \\
\hline Adipose & $1100 \pm 260$ & $851 \pm 140$ & $79 \pm 21$ & $78 \pm 31$ & N.D. & N.D. \\
\hline Liver & $11,000 \pm 1100$ & $10,000 \pm 1200$ & $1400 \pm 310$ & $990 \pm 180$ & $42 \pm 5.9$ & $42 \pm 5.7$ \\
\hline Kidney & $17,000 \pm 1100$ & $18,000 \pm 1400$ & $3900 \pm 620$ & $4000 \pm 570$ & $27 \pm 7.1$ & $17 \pm 2.0$ \\
\hline Brain & $17,000 \pm 4200$ & $12,000 \pm 560$ & $2400 \pm 790$ & $1300 \pm 140$ & $12 \pm 0.84$ & $4.7 \pm 1.4$ \\
\hline
\end{tabular}

N.D., Not determined.

$* P<0.05 ; * * P<0.01$.

amphetamine, and p-OHMA (Tables 2 and 3). Furthermore, our study identified Oct 3 as an important determinant of tissue uptake of and exposure to $p$-OHMA in salivary glands and skeletal muscle (Figs. 3 and 4). Our findings suggest that local tissue accumulation of methamphetamine and/or its metabolites may play a role in several of the reported peripheral toxicities of methamphetamine, and Oct3 can significantly impact tissue exposure to drugs and drug metabolites independently from their systemic exposure.

The abuse of methamphetamine is associated with several negative effects on health. Meth mouth, a condition characterized by xerostomia, rampant caries, and excessive tooth loss, represents a major health burden (Ravenel et al., 2012). The salivary glands, which synthesize and secrete saliva, play a vital role in oral and dental health (Humphrey and Williamson, 2001). Although the mechanisms leading to meth mouth are poorly understood, changes in saliva output and/or composition due to salivary glands dysfunction are believed to be the root cause (Hamamoto and Rhodus, 2009). A recent study indicated that xerostomia in methamphetamine abusers was not caused by a decreased saliva output but rather a change in the composition that may be caused by direct salivary glands toxicity (Ravenel et al., 2012). The decreased saliva $\mathrm{pH}$ and buffering capacity may indicate damage to the intercalated ductal cells responsible for bicarbonate salivary secretion (Whelton, 1996). We previously showed that OCT3 is highly expressed in both acini and ductal cells of salivary glands, which are involved in the modification and buffering of saliva (Whelton, 1996; Lee et al., 2014). We also demonstrated that Oct 3 mediates active uptake and high accumulation of metformin, an OCT3 substrate, in salivary glands in vivo (Lee et al., 2014). Recently, we identified that $p$-OHMA is a substrate of hOCT3 (Wagner et al., 2017) and confirmed it is a substrate of mOct3 (Fig. 1). These observations had led us to suspect that methamphetamine and/or metabolites may be actively transported into salivary glands by Oct3.

A novel finding of this study is that methamphetamine and metabolites are highly accumulated in salivary glands. Our data showed
A
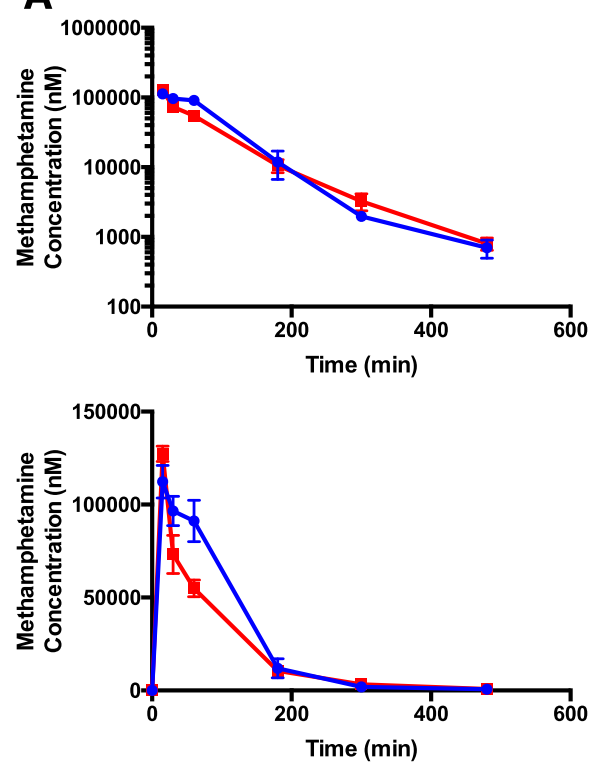

B
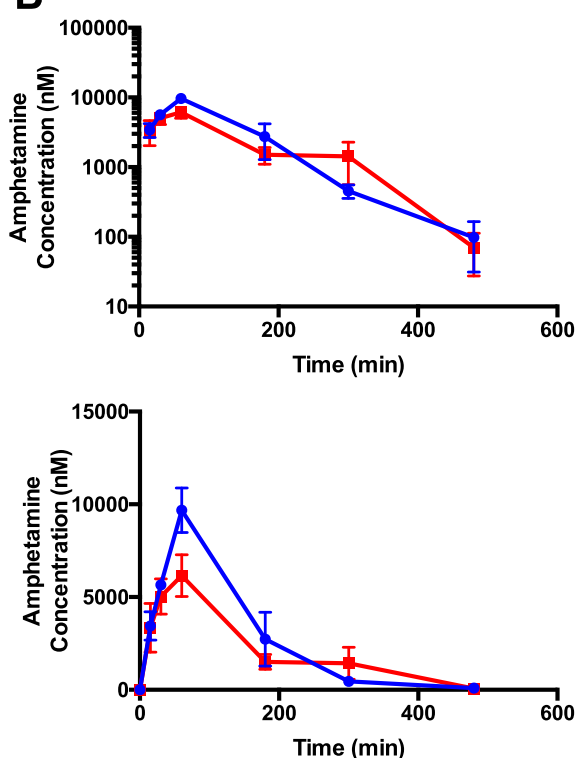

C
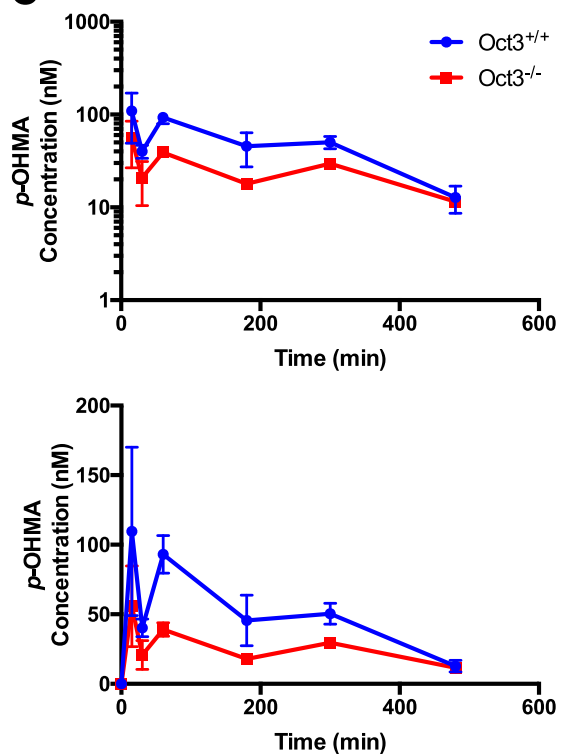

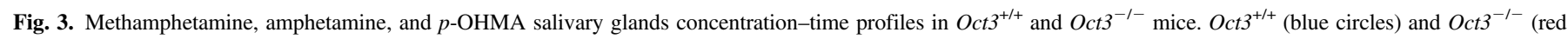

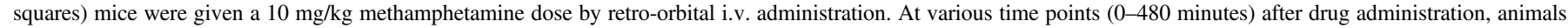

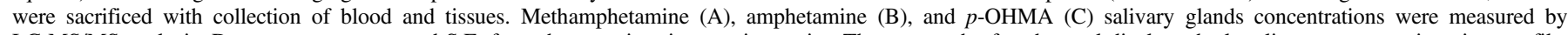

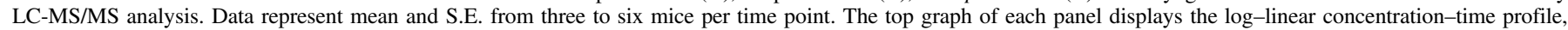
whereas the bottom graph displays the linear-linear profile. 
TABLE 3

Methamphetamine, amphetamine, and p-OHMA tissue to plasma $\mathrm{AUC}_{0-480}$ ratios in $O c t 3^{+/+}$and $O c t 3^{-/-}$mice

Data are presented as mean \pm S.E. Statistical significance was determined between genotypes for each analyte in each tissue using a permutation test with multiple comparisons corrected using the Bonferroni method, as detailed in Materials and Methods.

\begin{tabular}{|c|c|c|c|c|c|c|}
\hline \multirow{2}{*}{ Tissue } & \multicolumn{2}{|c|}{ Methamphetamine } & \multicolumn{2}{|c|}{ Amphetamine } & \multicolumn{2}{|c|}{$p$-OHMA } \\
\hline & $\operatorname{Oct}^{+/+}$ & $O c t 3^{-1-}$ & $\operatorname{Oct}^{+/+}$ & $O c t 3^{-1-}$ & $O c t 3^{+/+}$ & $O c t 3^{-1-}$ \\
\hline Salivary glands & $4.8 \pm 0.68$ & $4.4 \pm 0.48$ & $3.9 \pm 0.78$ & $4.0 \pm 0.89$ & $9.4 \pm 1.9$ & $4.5 \pm 0.89 *$ \\
\hline Muscle & $0.97 \pm 0.25$ & $1.2 \pm 0.16$ & $0.37 \pm 0.078$ & $0.63 \pm 0.15$ & $3.9 \pm 1.1$ & $0.91 \pm 0.34$ \\
\hline Heart & $1.9 \pm 0.29$ & $2.3 \pm 0.49$ & $1.4 \pm 0.22$ & $1.7 \pm 0.46$ & $2.9 \pm 0.75$ & $2.4 \pm 1.4$ \\
\hline Adipose & $0.44 \pm 0.11$ & $0.39 \pm 0.075$ & $0.24 \pm 0.071$ & $0.31 \pm 0.13$ & N.D. & N.D. \\
\hline Liver & $4.3 \pm 0.65$ & $4.8 \pm 0.71$ & $4.1 \pm 1.1$ & $3.9 \pm 0.91$ & $17 \pm 3.5$ & $16 \pm 3.7$ \\
\hline Kidney & $6.7 \pm 0.87$ & $8.3 \pm 1.0$ & $12 \pm 2.4$ & $16 \pm 3.3$ & $10 \pm 3.3$ & $6.6 \pm 1.4$ \\
\hline Brain & $6.4 \pm 1.8$ & $5.3 \pm 0.55$ & $7.1 \pm 2.5$ & $5.3 \pm 0.98$ & $4.8 \pm 0.86$ & $1.8 \pm 0.64$ \\
\hline
\end{tabular}

N.D., Not determined.

$* P<0.05$.

that in $\mathrm{Oct}^{+/+}$mice, methamphetamine, amphetamine, and $p$-OHMA accumulated in the salivary glands at concentrations several fold higher than plasma levels (Figs. 2 and 3; Table 2). The greatest level of accumulation was of $p$-OHMA, which had 9.4-fold higher exposure in the salivary glands than the plasma (Figs. 2 and 3; Table 3). This accumulation was mediated at least in part by Oct 3 , as demonstrated by the $50 \%$ reduction in the $p$-OHMA tissue AUC and partition ratio in salivary glands of $\mathrm{Oct}^{-1-}$ mice (Fig. 4; Table 3). The partition ratio of p-OHMA in $O c t 3^{-/-}$mice was similar to those observed for methamphetamine and amphetamine in both Cct $^{+/+}$and $O c t 3^{-/-}$mice (Table 3), which may be due to tissue binding, partitioning into subcellular compartments, or active uptake by an unidentified transporter. The high levels of accumulation of methamphetamine and metabolites in salivary glands suggest that one or more of these compounds may play a role in toxicity of salivary gland epithelial cells. As the toxicity of these compounds has not been examined, the main culprit(s) underlying salivary glands toxicity and meth mouth remains unclear. Future studies, such as chronic administration of each compound coupled with pathologic examination of salivary glands in $O c t 3^{+/+}$and $O c t 3^{-/-}$mice, could be helpful for further understanding the toxicological mechanisms leading to meth mouth.

Methamphetamine abuse is also associated with skeletal muscle toxicities. In severe cases, the breakdown of muscle (e.g., rhabdomyolysis) can lead to kidney failure and injury to other organs (Carvalho et al., 2012). Among the measured analytes, only $p$-OHMA is actively accumulated in skeletal muscle, with a partitioning ratio of 3.9 (Tables 2 and 3). Muscle

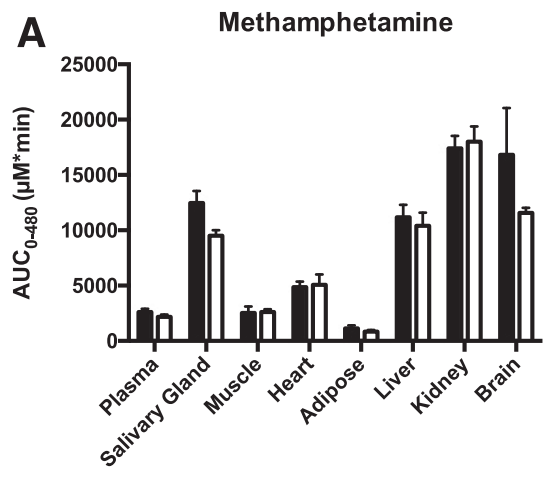

B

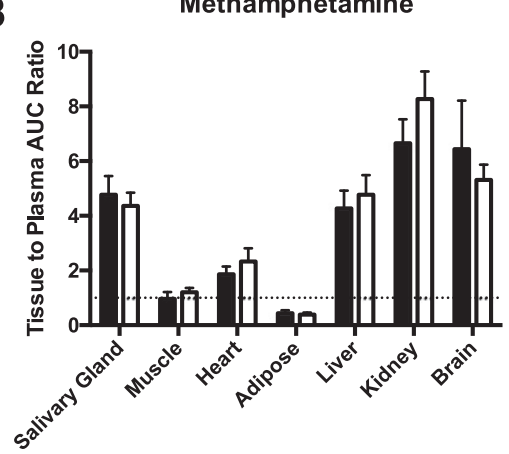

Amphetamine

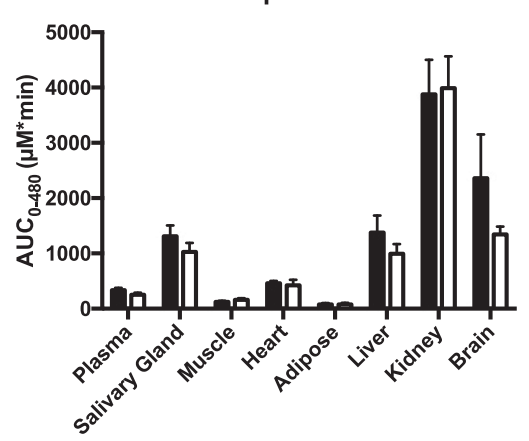

Amphetamine

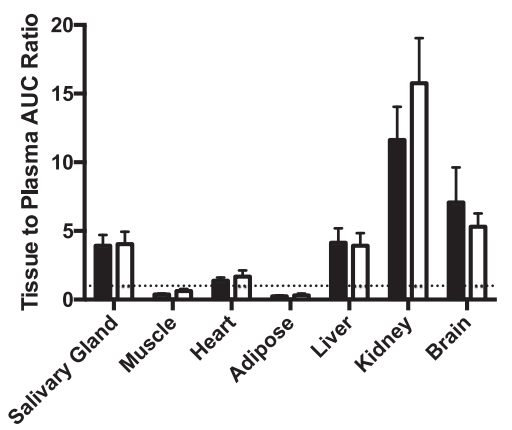

p-OHMA

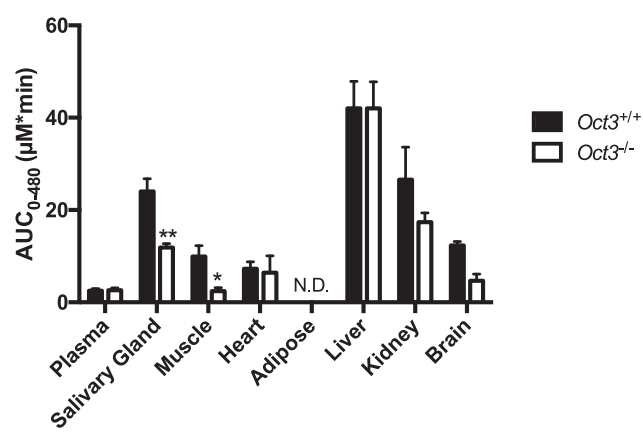

p-OHMA

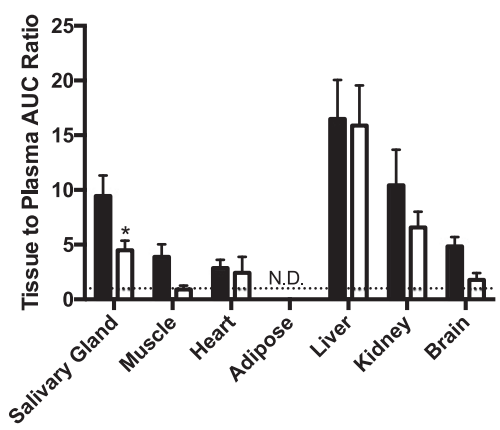

Fig. 4. Methamphetamine, amphetamine, and $p$-OHMA tissue AUC and AUC ratios in $O c t 3^{+/+}$and $O c t 3^{-/-}$mice. $O c t 3^{+/+}(\square)$ and $O c t 3^{-/-}$( $\square$ ) mice were given a $10 \mathrm{mg} / \mathrm{kg}$ methamphetamine dose by retro-orbital i.v. administration. At various time points (0-480 minutes) after drug administration, animals were sacrificed with collection of blood and tissues. Methamphetamine, amphetamine, and $p$-OHMA concentrations were measured by LC-MS/MS analysis, and the AUC (A) or tissue to plasma AUC ratio (B) was calculated. The dotted line denotes a ratio of one in (B). Data represent mean and S.E. estimated using a bootstrap approach from three to six mice per time point. Two-sided $P$ values were calculated using permutation tests for each analyte in each tissue, with multiple comparisons corrected using the Bonferroni method, as described under Materials and Methods $(* P<0.05 ; * P<0.01)$. 
p-OHMA exposure in $O c t 3^{-/-}$mice $(2.4 \mu \mathrm{M} \times \min )$ was significantly less than in $O c t 3^{+/+}$mice $(9.9 \mu \mathrm{M} \times \mathrm{min})$, indicating that Oct 3 may play a role in distribution to muscle (Fig. 4; Table 2). After normalizing to plasma AUC, the $p$-OHMA muscle partition ratio in $O c t 3^{-1-}$ mice was reduced by $\sim$ fourfold, although the difference between $O c t 3^{+/+}$and $\mathrm{Oct}^{-1-}$ mice did not reach statistical significance (Fig. 4; Table 3). Consistent with our observations, previous studies with metformin have provided evidence suggesting an in vivo role of Oct3 in muscle drug exposure in mice (Lee et al., 2014; Chen et al., 2015). Although we have no evidence to link $p$-OHMA with methamphetamine-induced muscle toxicities, our data revealed that $p$-OHMA is the only analyte that showed active accumulation in skeletal muscle after methamphetamine injection. Additional study is needed to elucidate whether OCT3 and $p$-OHMA are indeed involved in the peripheral toxicities of methamphetamine.

Oct3 is expressed in both neuronal and astroglial cells in multiple brain regions and is believed to act as part of the uptake- 2 system for clearing released monoamine neurotransmitters (Cui et al., 2009; Gasser et al., 2009; Amphoux et al., 2010; Duan and Wang, 2010; Klaassen and Aleksunes, 2010). Knockdown of Oct3 in mice was associated with altered methamphetamine-induced locomotor activity (Kitaichi et al., 2005). Although this study was unable to detect a significant difference in brain exposure of $p$-OHMA, the $\sim 2$.6-fold higher mean brain AUC and partition ratio of $p$-OHMA in $\mathrm{Oct}^{+/+}$mice (Tables 2 and 3) warrant future examination. It is still possible that Oct3 plays a role in brain disposition of $p$-OHMA and other organic cations. Currently, there is no functional data to support a role of Oct 3 in transporting organic cations at the blood-brain barrier (André et al., 2012). The seemingly higher AUC of $p$-OHMA in the $\mathrm{Oct}^{+/+}$mice may be due to Oct3-mediated substrate uptake and retention in neuronal and astroglial cells. Analysis of p-OHMA disposition in discrete brain regions with high Oct 3 expression and performing substrate uptake in isolated brain cells may provide additional clues.

Detailed methamphetamine and metabolite pharmacokinetics in humans who took methamphetamine recreationally are not available. Several opportunistic studies have measured plasma concentrations of methamphetamine, amphetamine, and $p$-OHMA in methamphetamine abusers, although the time of ingestion is often unknown (Römhild et al., 2003; Shima et al., 2008). The concentrations of methamphetamine, amphetamine, and $p$-OHMA observed in our study in mice were in the nanomolar to micromolar range, which are comparable to those reported in opportunistic studies in abusers (Römhild et al., 2003; Shima et al., 2008). Tissue concentrations and pharmacokinetics can be vastly different from those in the plasma. A previous human PET study using labeled $\left[{ }^{11} \mathrm{C}\right]$ methamphetamine identified the kidney, liver, and brain as high accumulation sites. However, because PET measures the total positron emission from the radionuclide, it could not differentiate between methamphetamine and metabolites containing the labeled carbon (Volkow et al., 2010). To our knowledge, the current study is the first to comprehensively determine methamphetamine and both primary metabolites (amphetamine and $p$-OHMA) pharmacokinetics in plasma and multiple tissues. Similar to the results from the human PET imaging study (Volkow et al., 2010), our study also found kidney, liver, and brain to be the major accumulation sites for methamphetamine. Furthermore, the metabolites (amphetamine and $p$-OHMA) are also highly accumulated in the kidney and liver (Fig. 4; Table 2).

We previously identified methamphetamine, amphetamine, and p-OHMA as substrates of human OCT2, suggesting renal accumulation and elimination may partially be driven by Oct 2 uptake (Wagner et al., 2017). In contrast to OCT2, tissue expression and in vivo studies consistently suggest that OCT3 plays a major role in peripheral tissue uptake but appears to be less important for systemic elimination (Lee et al., 2014; Chen et al., 2015; Wagner et al., 2016). Indeed, we observed little differences in plasma pharmacokinetics of methamphetamine and metabolites between $O c t 3^{+/+}$and $O c t 3^{-/-}$mice (Fig. 2; Tables 1 and 2) but substantial differences in disposition of $p$-OHMA in tissues known to highly express Oct3 (Figs. 3 and 4).

In summary, this study determined the pharmacokinetics, tissue exposure, and partition ratios of methamphetamine and major metabolites in various mouse tissues and investigated the impact of Oct 3 on tissue-specific accumulation of $p$-OHMA. Our data demonstrated salivary glands as a novel site of high accumulation of methamphetamine, amphetamine, and p-OHMA (Fig. 2; Table 2). Furthermore, our study identified Oct 3 as an important determinant of tissue uptake of and exposure to $p$-OHMA in salivary glands and skeletal muscle (Fig. 4; Tables 2 and 3). Our findings suggest that local tissue accumulation of methamphetamine and/or its metabolites may play a role in several of the reported peripheral toxicities of methamphetamine, and transporters can significantly impact tissue exposure to drugs and drug metabolites independently from their systemic exposure.

\section{Acknowledgments}

We thank Dr. Nina Isoherranen and Dr. Jenny Sager for expert assistance with the bioanalytical work. We are immensely grateful to Dr. Alfred Schinkel for the Oct3 knockout mice.

\section{Authorship Contributions}

Participated in research design: Wagner, Shen, Wang.

Conducted experiments: Wagner, Ahn.

Performed data analysis: Wagner, Shireman, Wang.

Wrote or contributed to the writing of the manuscript: Wagner, Shireman, Wang.

\section{References}

Amphoux A, Millan MJ, Cordi A, Bönisch H, Vialou V, Mannoury la Cour C, Dupuis DS, Giros $\mathrm{B}$, and Gautron S (2010) Inhibitory and facilitory actions of isocyanine derivatives at human and rat organic cation transporters 1,2 and 3: a comparison to human alpha 1- and alpha 2-adrenoceptor subtypes. Eur J Pharmacol 634:1-9.

André P, Saubaméa B, Cochois-Guégan V, Marie-Claire C, Cattelotte J, Smimova M, Schinkel AH, Scherrmann JM, and Cisternino S (2012) Transport of biogenic amine neurotransmitters at the mouse blood-retina and blood-brain barriers by uptake1 and uptake2. J Cereb Blood Flow Metab 32:1989-2001.

Carvalho M, Carmo H, Costa VM, Capela JP, Pontes H, Remião F, Carvalho F, and Bastos MdeL (2012) Toxicity of amphetamines: an update. Arch Toxicol 86:1167-1231.

Chen EC, Liang X, Yee SW, Geier EG, Stocker SL, Chen L, and Giacomini KM (2015) Targeted disruption of organic cation transporter 3 attenuates the pharmacologic response to metformin. Mol Pharmacol 88:75-83.

Copeland R (2000) Enzymes: A Practical Introduction to Structure, Mechanism, and Data Analysis, Wiley-VCH, New York.

Cui M, Aras R, Christian WV, Rappold PM, Hatwar M, Panza J, Jackson-Lewis V, Javitch JA, Ballatori N, Przedborski S, et al. (2009) The organic cation transporter-3 is a pivotal modulator of neurodegeneration in the nigrostriatal dopaminergic pathway. Proc Natl Acad Sci USA 106:8043-8048.

de la Torre R, Farré M, Navarro M, Pacifici R, Zuccaro P, and Pichini S (2004) Clinical pharmacokinetics of amfetamine and related substances: monitoring in conventional and nonconventional matrices. Clin Pharmacokinet 43:157-185.

Duan H and Wang J (2010) Selective transport of monoamine neurotransmitters by human plasma membrane monoamine transporter and organic cation transporter 3. J Pharmacol Exp Ther 335:743-753. Efron B and Tibshirani RJ (1994) An Introduction to the Bootstrap, CRC Press, Boca Raton, FL. Fowler JS, Kroll C, Ferrieri R, Alexoff D, Logan J, Dewey SL, Schiffer W, Schlyer D, Carter P, King P, et al. (2007) PET studies of d-methamphetamine pharmacokinetics in primates: comparison with 1-methamphetamine and (-)-cocaine. J Nucl Med 48:1724-1732.

Gasser PJ, Orchinik M, Raju I, and Lowry CA (2009) Distribution of organic cation transporter 3, a corticosterone-sensitive monoamine transporter, in the rat brain. J Comp Neurol 512:529-555. Hamamoto DT and Rhodus NL (2009) Methamphetamine abuse and dentistry. Oral Dis 15:27-37. Humphrey SP and Williamson RT (2001) A review of saliva: normal composition, flow, and function. J Prosthet Dent 85:162-169.

Jaki T and Wolfsegger MJ (2011) Estimation of pharmacokinetic parameters with the R package PK. Pharm Stat 10:284-288

Kim I, Oyler JM, Moolchan ET, Cone EJ, and Huestis MA (2004) Urinary pharmacokinetics of methamphetamine and its metabolite, amphetamine following controlled oral administration to humans. Ther Drug Monit 26:664-672.

Kitaichi K, Fukuda M, Nakayama H, Aoyama N, Ito Y, Fujimoto Y, Takagi K, Takagi K, and Hasegawa T (2005) Behavioral changes following antisense oligonucleotide-induced reduction of organic cation transporter-3 in mice. Neurosci Lett 382:195-200.

Klaassen CD and Aleksunes LM (2010) Xenobiotic, bile acid, and cholesterol transporters: function and regulation. Pharmacol Rev 62:1-96.

Koepsell H, Lips K, and Volk C (2007) Polyspecific organic cation transporters: structure, function, physiological roles, and biopharmaceutical implications. Pharm Res 24:1227-1251.

Lee N, Duan H, Hebert MF, Liang CJ, Rice KM, and Wang J (2014) Taste of a pill: organic cation transporter-3 (OCT3) mediates metformin accumulation and secretion in salivary glands. $J$ Biol Chem 289:27055-27064. 
Lee N, Hebert MF, Prasad B, Easterling TR, Kelly EJ, Unadkat JD, and Wang J (2013) Effect of gestational age on mRNA and protein expression of polyspecific organic cation transporters during pregnancy. Drug Metab Dispos 41:2225-2232.

Lin LY, Di Stefano EW, Schmitz DA, Hsu L, Ellis SW, Lennard MS, Tucker GT, and Cho AK (1997) Oxidation of methamphetamine and methylenedioxymethamphetamine by CYP2D6 Drug Metab Dispos 25:1059-1064.

Mager H and Göller G (1998) Resampling methods in sparse sampling situations in preclinical pharmacokinetic studies. J Pharm Sci 87:372-378

Panenka WJ, Procyshyn RM, Lecomte T, MacEwan GW, Flynn SW, Honer WG, and Barr AM (2013) Methamphetamine use: a comprehensive review of molecular, preclinical and clinical findings. Drug Alcohol Depend 129:167-179.

R Core Team (2017) R: A Language and Environment for Statistical Computing, R Foundation for Statistical Computing, Vienna, Austria.

RStudio Team (2012). RStudio: Integrated Development for R. RStudio, Inc., Boston, MA.

Ravenel MC, Salinas CF, Marlow NM, Slate EH, Evans ZP, and Miller PM (2012) Methamphetamine abuse and oral health: a pilot study of "meth mouth." Quintessence Int $\mathbf{4 3}$ 229-237.

Rivière GJ, Gentry WB, and Owens SM (2000) Disposition of methamphetamine and its metabolite amphetamine in brain and other tissues in rats after intravenous administration. $J$ Pharmacol Exp Ther 292:1042-1047.

Römhild W, Krause D, Bartels H, Ghanem A, Schöning R, and Wittig H (2003) LC-MS/MS analysis of pholedrine in a fatal intoxication case. Forensic Sci Int 133:101-106.

Schep LJ, Slaughter RJ, and Beasley DMG (2010) The clinical toxicology of metamfetamine. Clin Toxicol (Phila) 48:675-694.

Shaner JW, Kimmes N, Saini T, and Edwards P (2006) "Meth mouth": rampant caries in methamphetamine abusers. AIDS Patient Care STDS 20:146-150.

Shima N, Katagi M, Kamata H, Zaitsu K, Kamata T, Miki A, Tsuchihashi H, Sakuma T, and Nemoto N (2008) Conjugates of p-hydroxymethamphetamine and 4-hydroxy-3-methoxymethamphetamine in blood obtained from methamphetamine and 3,4-methylenedioxymethamphetamine users: analysis by LC-MS-MS. Forensic Toxicol 26:58-65.

Volkow ND (2013) Research Report Series: Methamphetamine, Rockville, MD.

Volkow ND, Fowler JS, Wang GJ, Shumay E, Telang F, Thanos PK, and Alexoff D (2010) Distribution and pharmacokinetics of methamphetamine in the human body: clinical implications. PLoS One 5:e15269.

Wagner DJ, Hu T, and Wang J (2016) Polyspecific organic cation transporters and their impact on drug intracellular levels and pharmacodynamics. Pharmacol Res 111:237-246.

Wagner DJ, Sager JE, Duan H, Isoherranen N, and Wang J (2017) Interaction and transport of methamphetamine and its primary metabolites by organic cation and multidrug and toxin extrusion transporters. Drug Metab Dispos 45:770-778.

Westfall PH and Young SS (1993) Resampling-Based Multiple Testing: Examples and Methods for p-Value Adjustment, John Wiley \& Sons, New York.

Whelton H (1996) Introduction: the anatomy and physiology of salivary glands, in Saliva and Oral Health pp 1-36, British Dental Association, London. Edgar, W.M. and O’Mullane, D.M., eds.

Wright SH (2005) Role of organic cation transporters in the renal handling of therapeutic agents and xenobiotics. Toxicol Appl Pharmacol 204:309-319.

Zhu HJ, Appel DI, Gründemann D, and Markowitz JS (2010) Interaction of organic cation transporter 3 (SLC22A3) and amphetamine. J Neurochem 114:142-149.

Zwart R, Verhaagh S, Buitelaar M, Popp-Snijders C, and Barlow DP (2001) Impaired activity of the extraneuronal monoamine transporter system known as uptake-2 in Orct3/Slc22a3-deficient mice. Mol Cell Biol 21:4188-4196.

Address correspondence to: Dr. Joanne Wang, Department of Pharmaceutics, University of Washington, H272J Health Sciences Building, Seattle, WA 981957610. E-mail: jowang@uw.edu 